\title{
Comparison of Ab Interno XEN Gelatin Stent vs Trabeculectomy with Mitomycin C: A Retrospective Study
}

\author{
Robert Sharpe ${ }^{1}$, Gina Pham², Peter Chang ${ }^{3}$
}

\begin{abstract}
Aim: To compare outcomes of ab interno XEN gelatin stent (Allergan, Dublin, Ireland) implantation vs trabeculectomy with mitomycin C (MMC). Materials and methods: A retrospective review was conducted of eyes that underwent standalone XEN implantation or trabeculectomy with MMC at a single institution from 2014 to 2019. Intraocular pressure (IOP), visual acuity, glaucoma medications, complications, and postoperative interventions were evaluated. The primary endpoint was the reduction in IOP at 6 months postoperatively. Secondary endpoints included the incidence of postoperative intervention and complications.

Results: One hundred and seventy-nine eyes were included who underwent XEN $(n=90)$ or trabeculectomy $(n=89)$. The mean age was 74.5 \pm 7.6 and $68.1 \pm 8.2$ years old for the XEN and trab groups, respectively $(p<0.001)$. Baseline IOP for XEN was $17.8 \pm 6.0 \mathrm{vs} 20.4 \pm 9.0 \mathrm{~mm} \mathrm{Hg}$ for the trab group $(p=0.03)$. At 6 months, mean IOP for XEN group was $13.5 \pm 5.9 \mathrm{~mm} \mathrm{Hg}$, representing a $24.1 \%$ IOP reduction from baseline ( $p$ $<0.001)$ while mean for trab group was $10.8 \pm 4.8$ representing a $47 \%$ IOP reduction from baseline $(p<0.001)$. The mean IOP was $2.7 \mathrm{~mm} \mathrm{Hg}$ lower in trab compared to the XEN group at 6 months $(p<0.003)$. The number of medications was reduced in both groups from $2.9 \pm 1.1$ and $3.1 \pm 0.9$ to $1.1 \pm 2.3$ and $0.8 \pm 1.4$ by 6 months postoperatively for XEN and trab groups, respectively $(p<0.001)$. Complication rates were low for both groups. The needling rate was $30 \%$ in XEN vs $7.9 \%$ in the trab group $(p<0.001)$, and $46.1 \%$ of eyes in the trab group underwent laser suture lysis. Additionally, IOP reduction and complication rates were similar following XEN in eyes receiving $<40$ or $\geq 40 \mu \mathrm{g}$ of MMC.

Conclusion: XEN implantation produces a substantial reduction in IOP with a favorable safety profile comparable to trabeculectomy. Careful postoperative bleb management is critical to obtain optimum outcomes, and higher MMC doses appear safe and may reduce needling rates. Clinical significance: This study confirms the safety and efficacy of XEN gelatin stent implantation in comparison to trabeculectomy with MMC. Keywords: Gelatin stent, Glaucoma surgery, Intraocular pressure, Trabeculectomy, XEN.

Journal of Current Glaucoma Practice (2020): 10.5005/jp-journals-10078-1287
\end{abstract}

\section{INTRODUCTION}

Glaucoma is currently the leading cause of irreversible blindness worldwide and continues to increase in prevalence. ${ }^{1}$ While a myriad of factors can result in glaucomatous vision loss, intraocular pressure (IOP) remains the only modifiable risk factor proven to limit progression. ${ }^{2}$ To lower IOP, initial treatment generally includes topical ocular antihypertensive medications or laser trabeculoplasty with traditional incisional glaucoma surgery (i.e., trabeculectomy and tube shunt implantation) reserved for more refractory disease due to higher risk profile. Trabeculectomy remains the gold standard glaucoma surgery as it typically results in lower IOP postoperatively than tube shunts but also carries a higher risk of complications. ${ }^{3,4}$

To address these safety concerns, minimally invasive glaucoma surgery (MIGS) has been under development in recent years. The XEN gelatin stent (Allergan, Dublin, Ireland), approved by the FDA in November 2017, was designed to be placed ab interno to connect aqueous to subconjunctival space. The gelatin stent is a 6-mm long crosslinked porcine collagen device with a $45-\mu \mathrm{m}$ inner diameter lumen. The fixed dimensions of the microstent provide a predictable resistance to outflow based on the Hagen-Poiseuille equation, which should limit postoperative hypotony. ${ }^{5}$

Multiple studies have reported the safety and efficacy of the XEN implant, suggesting an IOP reduction of 5.7-9.1 $\mathrm{mm} \mathrm{Hg}(23-40 \%)$. $^{6-13}$ However, few have directly compared XEN to trabeculectomy outcomes. ${ }^{7,10}$ In the retrospective study by Schlenker et al., ${ }^{7}$ the authors concluded that the XEN implant resulted in similar rates of failure as trabeculectomy. Marcos Parra et al. ${ }^{10}$ retrospectively reported a mean IOP reduction of $36.4 \%$ for XEN vs $36.2 \%$ for
${ }^{1-3}$ Department of Ophthalmology, Baylor College of Medicine, Houston, Texas, USA

Corresponding Author: Robert Sharpe, Department of Ophthalmology, Baylor College of Medicine, Houston, Texas, USA, Phone: +1 (910) 295-2100, e-mail: raseyemd19@gmail.com

How to cite this article: Sharpe R, Pham G, Chang P. Comparison of Ab Interno XEN Gelatin Stent vs Trabeculectomy with Mitomycin C: A Retrospective Study. J Curr Glaucoma Pract 2020;14(3):87-92.

Source of support: Nil

Conflict of interest: None

trabeculectomy at 12 months $(p=0.41)$. The authors concluded that although the two procedures produced similar IOP reductions, the XEN carried a more favorable safety profile.

In addition, although the dose-response relationship between the application of mitomycin C (MMC) and lower IOP has been well described for trabeculectomy, no studies have reported its role in XEN outcomes to date. ${ }^{14-16}$ This report aims to compare outcomes of primary, standalone XEN vs trabeculectomy with MMC with respect to IOP and medication reduction as well as complications and postoperative intervention rates. Additionally, the effect of MMC dose on XEN outcomes will be explored.

\section{Materials and Methods}

This retrospective cohort study evaluated eyes that underwent XEN gelatin stent implantation at a single institution between May

(c) Jaypee Brothers Medical Publishers. 2020 Open Access This article is distributed under the terms of the Creative Commons Attribution 4.0 International License (https://creativecommons.org/licenses/by-nc/4.0/), which permits unrestricted use, distribution, and non-commercial reproduction in anymedium, provided you give appropriate credit to the original author(s) and the source, provide a link to the Creative Commons license, and indicate if changes were made. The Creative Commons Public Domain Dedication waiver (http://creativecommons.org/publicdomain/zero/1.0/) applies to the data made available in this article, unless otherwise stated. 
2017 and January 2019. Patients were identified by surgical logs of a single surgeon and clinical data were extracted from the electronic medical record. All research activities were approved by the Baylor College of Medicine Institutional Review Board and conducted in accordance with the Declaration of Helsinki.

Eyes were included who underwent standalone XEN implantation and were followed for up to 6 months postoperatively. Demographics and baseline characteristics such as IOP, visual acuity, number of glaucoma medications, glaucoma diagnosis, and prior ocular procedures were recorded. Postoperative course including complications and interventions were documented over the 6 months. Exclusion criteria consisted of prior incisional glaucoma surgery, combined cataract-XEN surgery, and loss to follow-up before 1 month. In addition, a control group of eyes receiving a standard trabeculectomy with MMC, performed by the same surgeon, was included. These patients were collected from 2014 to 2018 but otherwise followed the same inclusion and exclusion criteria.

XEN gelatin stent implantation was performed ab interno under topical anesthesia.

The superonasal target site for the gelatin stent was identified 3-mm posterior to the limbus. Subtenon injection of MMC (10-60 $\mu \mathrm{g}$ ) was performed superiorly and massaged posteriorly toward the superior fornix. A superotemporal corneal paracentesis was created, and Healon GV (Johnson and Johnson, Santa Ana, CA, USA) was injected into the anterior chamber. A biplanar $1.8-\mathrm{mm}$ inferotemporal corneal wound was created, and the XEN injector, pre-loaded with the XEN 45 Gel Stent, was inserted and advanced toward the superonasal iridocorneal angle. Intraoperative gonioscopy was used to guide the XEN injector tip slightly anterior to the trabecular meshwork. Then, the gelatin stent was injected and confirmed to exit the sclera at the pre-determined location. Healon GV was removed from the anterior chamber by instilling a balanced salt solution through the corneal wound followed by an injection of $0.4 \mathrm{mg}$ of dexamethasone sodium phosphate. After the case, wounds were hydrated and confirmed to be watertight.

The trabeculectomy was performed via a limbus-based approach in all cases. Retrobulbar anesthesia was achieved with a $50: 50$ mixture of $2 \%$ lidocaine without epinephrine and $0.5 \%$ bupivacaine with hyaluronidase. A 7-0 Vicryl (Johnson and Johnson, Santa Ana, CA, USA) superior corneal traction suture was placed to infraduct the globe. A small conjunctival incision to allow subtenon infusion of MMC (10-40 $\mu \mathrm{g})$ superiorly and massaged superiorly. Superiorly, a peritomy and posterior dissection were performed using blunt scissors. Hemostasis was achieved using wet-field bipolar cautery. A partial-thickness, trapezoidal scleral flap, hinged at the limbus, was fashioned using a 15-degree blade and \#67 Beaver blade (Beaver-Visitec International, Waltham, MA, USA). A corneal paracentesis was created followed by entry to the anterior chamber at the anterior aspect of the scleral flap using a 15-degree blade. Posterior-lip sclerotomy was performed with Luntz-Dodick trabeculectomy punch (Katena, Denville, NJ, USA), followed by a peripheral iridectomy. A single interrupted 10-0 nylon suture was used to close the scleral flap at each corner with tension adjusted to achieve the appropriate IOP. Conjunctiva was reapproximated to the limbus using a 10-0 nylon in a horizontal mattress fashion at the midpoint and an 8-0 Vicryl vertical mattress suture at each end with running closure of the relaxing incisions. Watertight wound closure was confirmed and $0.4 \mathrm{mg}$ of dexamethasone sodium phosphate was injected into the anterior chamber followed by removal of the traction suture.

The primary outcome measure was IOP reduction from baseline at 6 months postoperatively. Secondary outcome measures included rates of "success" and complications. Success was defined as a $20 \%$ IOP reduction from baseline. Additionally, MMC dosing was evaluated in the XEN group.

Data were recorded in Microsoft Excel for Mac (Microsoft, Redmond, WA, USA), and statistical analysis was performed with SPSS (IBM, Armonk, NY, USA). Means with standard deviations (SD) were calculated for IOP, visual acuity, and several medications at all time points. Snellen visual acuity was converted to logMAR for statistical comparison. Student's $t$-test and Mann-Whitney $U$ tests were used to compare continuous variables. Categorical variables were compared using Chi-square and Fisher's exact tests. Statistical significance was set at $p<0.05$.

\section{Results}

A total of 179 eyes were included with 90 in the XEN group (XEN) and 89 in the trabeculectomy group (trab). Baseline demographic characteristics are summarized in Table 1. Mean age was about 6 years older in the XEN group at $74.5 \pm 7.6$ vs $68.1 \pm 8.2$ years old for the XEN and trab eyes, respectively $(p<0.001)$. In XEN group, $61 \%$ of patients were female vs $40 \%$ in trab group $(p=0.03)$. In addition, there was a higher proportion of white race in the XEN group at 80 vs $38 \%$ in the trab group $(p<0.001)$. The type of glaucoma diagnosis was similar between groups with the primary open-angle being most common in both $(p>0.28)$. A higher proportion of patients in the XEN group had undergone previous cataract surgery (62\%) vs those in the trab group $(36 \%)(p<0.001)$.

Baseline IOP was lower on average for the XEN group averaging $17.8 \pm 6.0$ and $20.4 \pm 9.0 \mathrm{~mm} \mathrm{Hg}$ for XEN and trab groups, respectively ( $p=0.03$ ). Both groups were on a similar number of ocular antihypertensive medications at baseline with a mean of $2.9 \pm 1.1$ and $3.1 \pm 0.9$ medications for XEN and trab groups, respectively $(p=0.24)$. Ocular baseline parameters are summarized in Table 2.

At 6 months, both groups experienced significant reductions in IOP on average as summarized in Table 3. The mean IOP for the XEN group was $13.5 \pm 5.9 \mathrm{~mm} \mathrm{Hg}$ representing a $24.1 \%$ IOP reduction from baseline $(p<0.001)$. The mean IOP at 6 months for the trab group was $10.8 \pm 4.8$ representing a $47 \%$ IOP reduction from baseline $(p<0.001)$. At 6 months, the trab group exhibited a lower mean IOP compared to XEN by $2.7 \mathrm{~mm} \mathrm{Hg}(p<0.003)$. The IOP curve, at preoperative visit and postoperative visits out to 6 months are displayed in Figure 1. Regarding surgical "success" rates as defined by at least a $20 \%$ reduction in IOP from baseline at 6 months, $56 \%$ of the XEN group vs $78 \%$ of the trab group met this criterion.

The number of ocular antihypertensive medications was reduced in both groups at 6 months to $1.1 \pm 2.3$ and $0.8 \pm 1.4$ for XEN and trab groups, respectively $(p<0.001)$ as shown in Table 3. At 6 months, the number of medications used was similar between the two groups $(p=0.39)$. Visual acuity was similar between the two groups both preoperatively and postoperatively and did not change significantly at 6 months ( $p>0.3$ ).

Complication rates were overall low and similar between the two procedures as shown in Table 4. In XEN group, 30\% of eyes underwent needling vs $7.9 \%$ in trab group $(p<0.001)$. However, $46.1 \%$ of eyes in the trab group underwent laser suture lysis to 
Table 1: Baseline demographic characteristics for XEN and trab groups

\begin{tabular}{|c|c|c|c|c|c|c|c|}
\hline & & \multicolumn{2}{|c|}{ XEN } & \multicolumn{2}{|c|}{ Trab } & \multirow[b]{2}{*}{$p$ value } & \\
\hline & & Mean & $S D$ & Mean & $S D$ & & \\
\hline$n$ & & 90 & & 89 & & & \\
\hline Age (years) & & 74.5 & 7.6 & 68.1 & 8.2 & $<0.001$ & $t$-test \\
\hline \multirow[t]{2}{*}{ Sex (female) } & $n$ & 55 & & 36 & & & \\
\hline & $\%$ & $61.1 \%$ & & $40.4 \%$ & & 0.03 & Fisher's exact \\
\hline \multirow[t]{5}{*}{ Race } & White & 72 & & 34 & & $<0.001$ & Fisher's exact \\
\hline & Black & 4 & & 29 & & $<0.001$ & Fisher's exact \\
\hline & Asian & 5 & & 15 & & 0.02 & Fisher's exact \\
\hline & Hispanic & 9 & & 9 & & 1 & Fisher's exact \\
\hline & Native American & 0 & & 1 & & 0.50 & Fisher's exact \\
\hline \multirow[t]{5}{*}{ Glaucoma diagnosis } & POAG & 74 & & 76 & & 0.69 & Fisher's exact \\
\hline & LTG & 10 & & 5 & & 0.28 & Fisher's exact \\
\hline & PXF & 3 & & 3 & & 1 & Fisher's exact \\
\hline & Pigmentary & 1 & & 2 & & 0.62 & Fisher's exact \\
\hline & Secondary & 2 & & 3 & & 0.68 & Fisher's exact \\
\hline \multirow[t]{2}{*}{ Prior eye surgery } & Cataract surgery & 56 & & 32 & & $<0.001$ & Fisher's exact \\
\hline & PPV & 5 & & 5 & & 1 & Fisher's exact \\
\hline
\end{tabular}

Table 2: Baseline ocular parameters for XEN and trab groups

\begin{tabular}{|c|c|c|c|c|c|c|}
\hline & & \multicolumn{2}{|c|}{$X E N$} & \multicolumn{2}{|c|}{ Trab } & \multirow[b]{2}{*}{$p$ value } \\
\hline & & Mean & $S D$ & Mean & $S D$ & \\
\hline \multicolumn{7}{|l|}{ Baseline } \\
\hline & Vision & 0.3 & 0.4 & 0.3 & 0.5 & 0.58 \\
\hline & IOP & 17.8 & 6.0 & 20.4 & 9.0 & 0.03 \\
\hline & \# meds & 2.9 & 1.1 & 3.1 & 0.9 & 0.24 \\
\hline
\end{tabular}

Table 3: Comparison of baseline and 6-month ocular parameters for XEN and trab groups

\begin{tabular}{|c|c|c|c|c|c|c|}
\hline & & \multicolumn{2}{|c|}{ Baseline } & \multicolumn{2}{|c|}{6 months } & \multirow[b]{2}{*}{$p$ value } \\
\hline & & Mean & $S D$ & Mean & $S D$ & \\
\hline \multicolumn{7}{|l|}{ XEN } \\
\hline & Vision & 0.3 & 0.4 & 0.3 & 0.4 & 0.3 \\
\hline & IOP & 17.8 & 6.0 & 13.5 & 5.9 & $<0.001$ \\
\hline & \# meds & 2.9 & 1.1 & 1.1 & 2.3 & $<0.001$ \\
\hline \multicolumn{7}{|l|}{ Trab } \\
\hline & Vision & 0.3 & 0.5 & 0.3 & 0.3 & 0.63 \\
\hline & $\mathrm{IOP}$ & 20.4 & 9.0 & 10.8 & 4.8 & $<0.001$ \\
\hline & \# meds & 3.1 & 0.9 & 0.8 & 1.4 & $<0.001$ \\
\hline
\end{tabular}

control IOP. The trab group also exhibited a higher rate of bleb leaks at 7.9 vs $1.1 \%$ with the XEN group, respectively $(p=0.03)$. The hyphema rate of the XEN group was $13.3 \%$ compared to $2.2 \%$ in the trab group $(p<0.01)$, but all cases were transient and resolved spontaneously. Rates of other serious complications were similar between the two groups.

Eyes in the XEN group were then divided based on MMC dose into those receiving $<40$ or $\geq 40 \mu \mathrm{g}$ of MMC. Preoperative IOP was similar between the two groups at $18.2 \pm 6.1$ and $17.5 \pm 5.9$ for the $<40 \mu \mathrm{g} M M C$ and $\geq 40 \mu \mathrm{g}$ MMC groups, respectively ( $p=0.59$ ). The postoperative IOP curve is shown in Figure 2 . The needling rate was 29.5 and $30.4 \%$ for the $<40 \mu \mathrm{g} M M C$ and $\geq 40 \mu \mathrm{g}$ MMC groups, respectively $(p=0.99)$.

\section{Discussion}

In the era of MIGS, a favorable safety profile is one of the primary attributes of emerging glaucoma surgical techniques. The fixed dimensions and biocompatible properties of the XEN device were specifically designed to reduce rates of complications, especially hypotony, in eyes undergoing glaucoma filtering surgery. The general mechanism of subconjunctival filtration of aqueous from the anterior chamber likens the XEN more to traditional trabeculectomy than to typical MIGS procedures, which often employ conventional outflow pathways. Therefore, to fully understand the role of the XEN device, comparisons between it and trabeculectomy are required. 


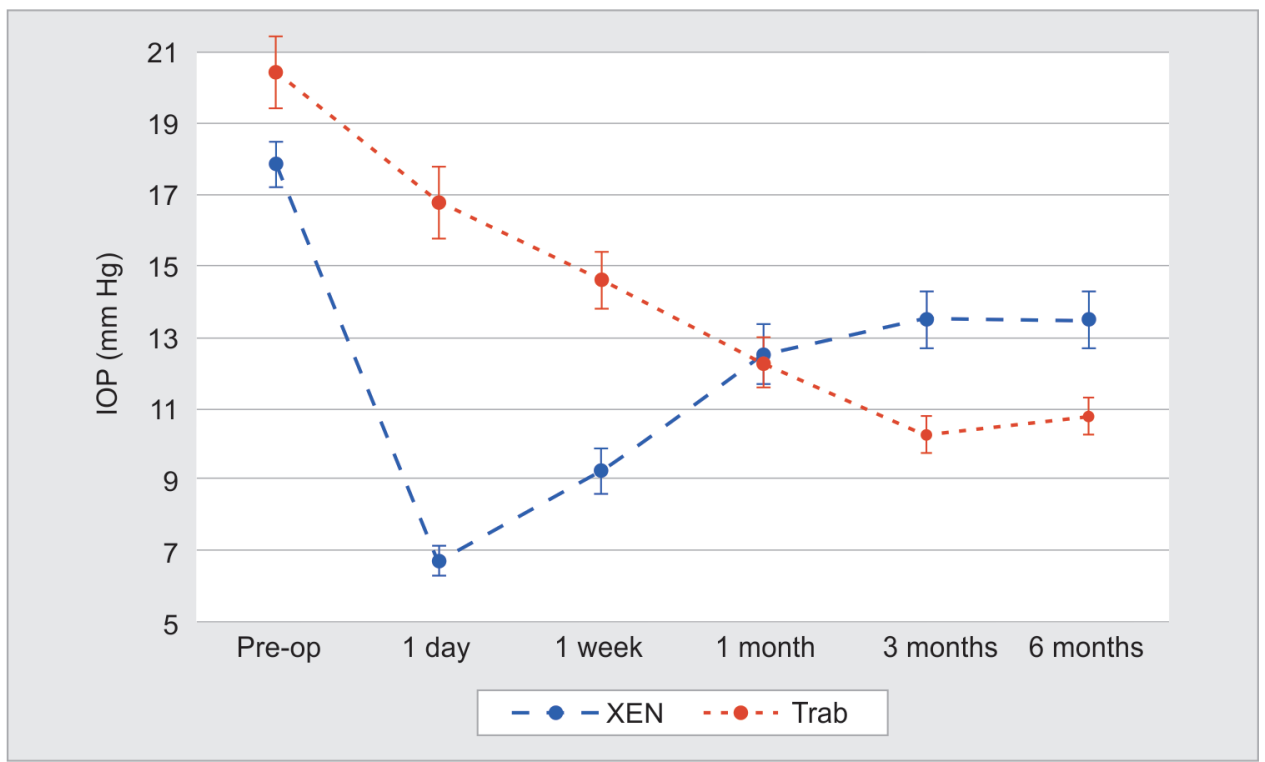

Fig. 1: Mean IOP curve preoperatively to 6 months postoperatively for XEN and trab groups

Table 4: Complication profiles following XEN and trab surgery

\begin{tabular}{|c|c|c|c|c|c|}
\hline & \multicolumn{2}{|c|}{$X E N$} & \multicolumn{2}{|c|}{ Trab } & \multirow[b]{2}{*}{$p$ value } \\
\hline & $N$ & $\%$ & $N$ & $\%$ & \\
\hline Needling & 27 & 30.0 & 7 & 7.9 & $<0.001$ \\
\hline LSL & $\mathrm{n} / \mathrm{a}$ & $n / a$ & 41 & 46.1 & $n / a$ \\
\hline $\begin{array}{l}\text { Additional glaucoma } \\
\text { surgery }\end{array}$ & 7 & 7.8 & 4 & 4.5 & 0.54 \\
\hline Bleb leak & 1 & 1.1 & 7 & 7.9 & 0.03 \\
\hline Shallow/flat AC & 1 & 1.1 & 3 & 3.4 & 0.37 \\
\hline Hyphema & 12 & 13.3 & 2 & 2.2 & 0.01 \\
\hline Blebitis & 1 & 1.1 & 1 & 1.1 & 1 \\
\hline Endophthalmitis & 0 & 0.0 & 1 & 1.1 & 0.5 \\
\hline
\end{tabular}

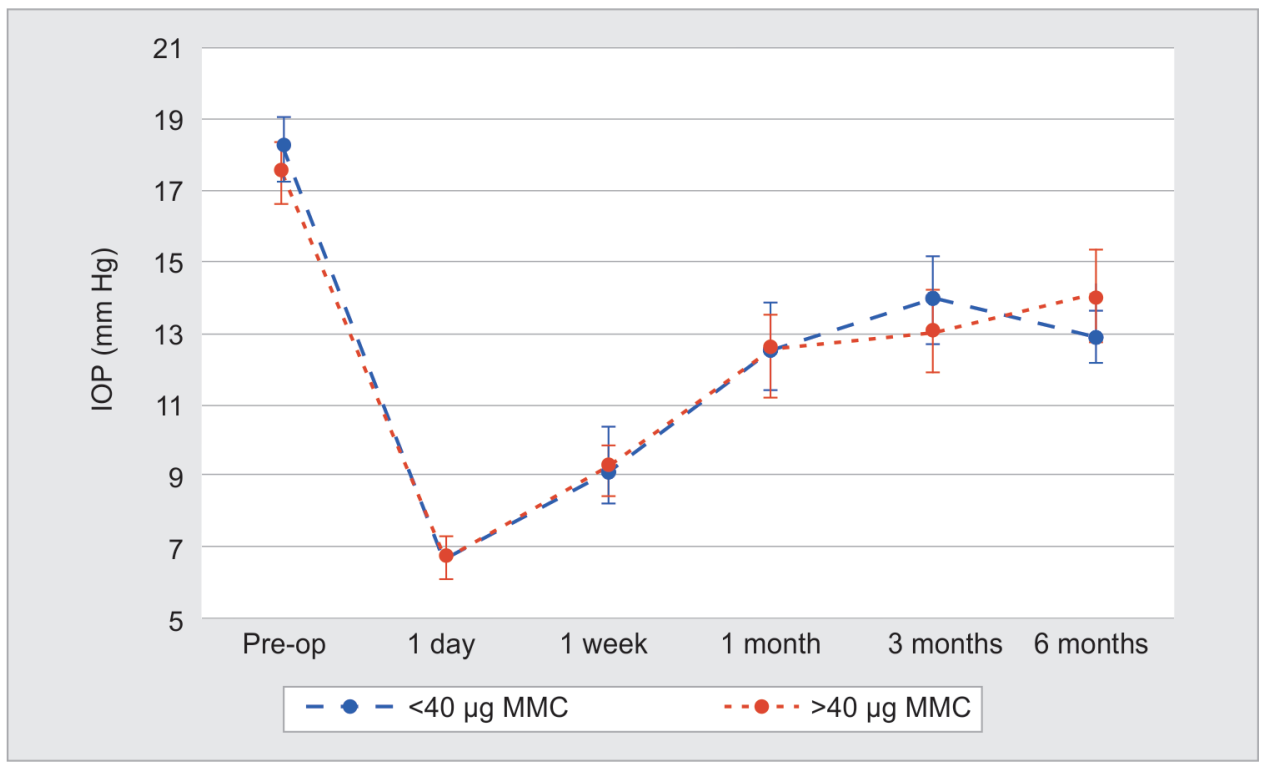

Fig. 2: Mean IOP curve preoperatively to 6 months postoperatively following XEN based on mitomycin C dose 
However, few studies have directly compared XEN to trabeculectomy outcomes., ${ }^{7,10}$ In the retrospective study by Schlenker et al., ${ }^{7}$ the authors concluded that the XEN implant resulted in similar rates of failure as trabeculectomy. Marcos Parra et al. $^{10}$ retrospectively reported a mean IOP reduction of $36.4 \%$ for XEN vs $36.2 \%$ for trabeculectomy at 12 months ( $p=0.41$ ). The authors concluded that although the two procedures produced similar IOP reductions, the XEN carried a more favorable safety profile. The results of our retrospective cohort study add to the limited current body of data that both XEN and trabeculectomy with MMC produce significant IOP reductions with lower ocular antihypertensive medication usage at 6 months postoperatively in patients with refractory glaucoma.

In our study, the mean IOP at 6 months was lower for the trabeculectomy group by $2.7 \mathrm{~mm} \mathrm{Hg}(p<0.003)$ compared to the XEN group. This finding suggests that while both procedures produce marked IOP reductions, there may be a slight IOP advantage for the trabeculectomy in this patient cohort. Both groups exhibited significant reductions in medication usage following surgery, and there was no statistically significant difference in the number of medications used at 6 months between the groups.

Prior reports have indicated a mean IOP reduction of 5.7-9.1 $\mathrm{mm} \mathrm{Hg}(23-40 \%)$ following XEN implantation. ${ }^{6-13}$ These results are overall consistent with the present study which showed an average of $4.3 \mathrm{~mm} \mathrm{Hg}(24.1 \%)$ at 6 months postoperatively. The reason for the lower absolute IOP reduction in this study is likely due to a lower mean baseline IOP of $17.8 \mathrm{~mm} \mathrm{Hg}$ in eyes receiving XEN in this study compared to a mean baseline of $22.9 \mathrm{~mm} \mathrm{Hg}$ (range $21.7-25.1 \mathrm{~mm}$ $\mathrm{Hg}$ ) in other studies. . $^{6,10-12}$

Overall, the rates of complications were similar and low between XEN and trabeculectomy. The main differences were the frequency of hyphema and blebitis. The most common complication in the XEN group was hyphema occurring in 13\% of eyes vs $2.2 \%$ in the trabeculectomy group $(p=0.01)$. Importantly, this was a self-limited process and resolved spontaneously in all cases. Eyes with hyphema did not fare worse than those without. In the trabeculectomy group, the most common complication was bleb leak, which occurred in $7.9 \%$ of cases vs only $1 \%$ in the XEN group ( $p=0.03$ ). The need for additional glaucoma surgery was low and similar between groups, occurring in 7.8 and $4.5 \%$ of eyes undergoing XEN and trabeculectomy, respectively $(p>0.5)$. One eye in each group experienced blebitis, but in the trabeculectomy group, that eye proceeded to develop endophthalmitis.

The need for postoperative interventions was different between the two procedures. Laser suture lysis was the most common procedure performed on trabeculectomy eyes and was required in $46.1 \%$ of cases. While the need for suture lysis is not considered a complication as it is an expected method to titrate aqueous flow following trabeculectomy, the necessity of an additional intervention in the clinic to obtain the desired postoperative outcome is important to consider.

While there are no flap sutures to laser following XEN implantation, needling of the bleb is the most common intervention employed to restore flow postoperatively. While the indications for needling to optimize bleb function with the XEN are not uniform among studies, rates of XEN needling have typically fallen between 20 and $44.1 \% .^{7-10,13,17}$ In our study, 30\% of eyes receiving XEN underwent postoperative bleb needling vs $7.9 \%$ of eyes with trabeculectomy $(p<0.01)$, which is consistent with prior reports. The high rate of bleb manipulation through needling following XEN stems from the fact that there are no other titratable features of this device to alter the flow. In addition, there appears to be a tendency for Tenon's capsule to adhere to the device and can obstruct flow at the distal end, especially if the XEN is not placed precisely in the subconjunctival space. This low flow state can predispose the area to subconjunctival scarring and a subsequent recalcitrant IOP rise. ${ }^{9,18}$ Therefore, an aggressive approach to bleb management is typically employed to optimize longevity similar to postoperative trabeculectomy management.

The dose of MMC used in this study was significantly higher on average in the XEN group compared to trabeculectomy. This difference stems from an emerging practice pattern of using higher MMC doses with the XEN to reduce needling rates and improve IOP outcomes. Higher doses of MMC have long been associated with lower IOP in trabeculectomy but often also carry increased rates of complications. ${ }^{14,15,19}$ The initial studies during FDA approval often employed conjunctival cutdown and application of MMC using soaked sponges, ${ }^{6,12,20}$ making extrapolating the appropriate dose for subconjunctival injection difficult. Most studies employing subconjunctival infusion report a range of 0.1 to $0.2 \mathrm{~cm}^{3}$ of 0.1 to $0.2 \mathrm{mg} / \mathrm{mL}$ (i.e., $10-40 \mu \mathrm{g}$ ) of MMC. . $^{70,13,21}$

However, given the reduced concern of hypotony due to the XEN's fixed lumen size and minimal need for wound healing with the injection technique, higher doses of MMC can theoretically be employed without the typical increase in complications. Therefore, there has been a gradual trend to increase the dose of MMC in XEN surgery to reduce needling rates. In our study, there was no significant difference in complications between eyes receiving $<40$ or $\geq 40 \mu \mathrm{g}$ MMC. The IOP in these two groups was similar over 6 months postoperatively suggesting that increasing the dose in higher risk eyes can produce comparable results to lower risk eyes. This is the first report to our knowledge on MMC dosing effects on XEN outcomes.

The main limitations of this study relate to its retrospective nature and patient selection. The retrospective design always carries the risk of selection bias, the variability of treatment, and loss of follow-up. We attempted to address selection bias by including all XEN surgeries performed that met inclusion criteria. However, the results suggest that certain demographic features were not evenly matched between XEN and trabeculectomy groups, especially with respect to age, race, MMC dose, and baseline IOP, which limits generalizability from intergroup comparisons. The variability in surgical technique and postoperative management was controlled by including cases from only a single surgeon.

\section{Conclusion}

The XEN gelatin implant produces a substantial reduction in IOP with a favorable safety profile, but the postoperative IOP may not be as low as trabeculectomy. Importantly, both procedures require careful postoperative bleb management to optimize outcomes, and for the XEN, higher MMC doses appear safe and may reduce needling rates.

\section{References}

1. Tham YC, Li X, Wong TY, et al. Global prevalence of glaucoma and projections of glaucoma burden through 2040: a systematic review and meta-analysis. Ophthalmology 2014;121(11):2081-2090. DOI: 10.1016/j.ophtha.2014.05.013.

2. Heijl A, Leske MC, Bengtsson B, et al. Reduction of intraocular pressure and glaucoma progression: results from the early manifest 
glaucoma trial. Arch Ophthalmol 2002;120(10):1268-1279. DOI: 10.1001/archopht.120.10.1268.

3. Gedde SJ, Feuer WJ, Shi W, et al. Treatment outcomes in the primary tube versus trabeculectomy study after 1 year of follow-up. Ophthalmology 2018;125(5):650-663. DOI: 10.1016/j. ophtha.2018.02.003.

4. Gedde SJ, Herndon LW, Brandt JD, et al. Postoperative complications in the tube versus trabeculectomy (TVT) study during five years of follow-up. Am J Ophthalmol 2012;153(5):804-814.e1. DOI: 10.1016/j. ajo.2011.10.024.

5. Lewis RA. Ab interno approach to the subconjunctival space using a collagen glaucoma stent. J Cataract Refract Surg 2014;40(8):13011306. DOI: 10.1016/j.jcrs.2014.01.032.

6. Grover DS, Flynn WJ, Bashford KP, et al. Performance and safety of a new ab interno gelatin stent in refractory glaucoma at 12 months. Am J Ophthalmol 2017;183:25-36. DOI: 10.1016/j.ajo.2017.07.023.

7. Schlenker MB, Gulamhusein H,Conrad-Hengerer I, et al. Efficacy, safety, and risk factors for failure of standalone ab interno gelatin microstent implantation versus standalone trabeculectomy. Ophthalmology 2017;124(11):1579-1588. DOI: 10.1016/j.ophtha.2017.05.004.

8. Heidinger A, Schwab C, Lindner E, et al. A retrospective study of 199 Xen45 stent Implantations from 2014 to 2016. J Glaucoma 2019;28(1):75-79. DOI: 10.1097/IJG.0000000000001122.

9. Karimi A, Lindfield $D$, Turnbull A, et al. A multi-centre interventional case series of $259 \mathrm{ab}$-interno XEN gel implants for glaucoma, with and without combined cataract surgery. Eye (Lond) 2019;33(3):469-477. DOI: 10.1038/s41433-018-0243-8.

10. Marcos Parra MT, Salinas Lopez JA, Lopez Grau NS, et al. XEN implant device versus trabeculectomy, either alone or in combination with phacoemulsification, in open-angle glaucoma patients. Graefes Arch Clin Exp Ophthalmol 2019;257(8):1741-1750. DOI: 10.1007/s00417-01904341-y.

11. Lenzhofer M, Kersten-Gomez I, Sheybani A, et al. Four-year results of a minimally invasive transscleral glaucoma gel stent implantation in a prospective multi-centre study. Clin Exp Ophthalmol 2019;47(5): 581-587. DOI: 10.1111/ceo.13463.
12. Reitsamer H, Sng C, Vera V, et al. Two-year results of a multicenter study of the ab interno gelatin implant in medically uncontrolled primary open-angle glaucoma. Graefes Arch Clin Exp Ophthalmol 2019;257(5):983-996. DOI: 10.1007/s00417-019-04251-z.

13. Smith M, Charles R, Abdel-Hay A, et al. 1-Year outcomes of the Xen 45 glaucoma implant. Eye (Lond) 2019;33(5):761-766. DOI: 10.1038/ s41433-018-0310-1.

14. Mietz $\mathrm{H}$, Krieglstein GK. Three-year follow-up of trabeculectomies performed with different concentrations of mitomycin-C. Ophthalmic Surg Lasers 1998;29(8):628-634.

15. Sanders SP, Cantor LB, Dobler AA, et al. Mitomycin C in higher risk trabeculectomy: a prospective comparison of 0.2- to $0.4-\mathrm{mg} / \mathrm{cc}$ doses. J Glaucoma 1999;8(3):193-198. DOI: 10.1097/00061198-19990600000008.

16. Kupin $\mathrm{TH}$, Juzych MS, Shin $\mathrm{DH}$, et al. Adjunctive mitomycin C in primary trabeculectomy in phakic eyes. Am J Ophthalmol 1995;119(1):30-39. DOI: 10.1016/S0002-9394(14)73810-3.

17. Gillmann K, Bravetti GE, Mermoud A, et al. XEN gel stent in pseudoexfoliative glaucoma: two-year results of a prospective evaluation. J Glaucoma 2019;28(8):676-684. DOI: 10.1097/ IJG.0000000000001295.

18. Tan SZ, Walkden A, Au L. One-year result of XEN45 implant for glaucoma: efficacy, safety, and postoperative management. Eye (Lond) 2018;32(2):324-332. DOI: 10.1038/eye. 2017.162.

19. Kitazawa $Y$, Suemori-Matsushita $H$, Yamamoto T, et al. Low-dose and high-dose mitomycin trabeculectomy as an initial surgery in primary open-angle glaucoma. Ophthalmology 1993;100(11):1624-1628. DOI: 10.1016/S0161-6420(93)31426-0.

20. Sheybani A, Dick HB, Ahmed II. Early clinical results of a novel ab interno gel stent for the surgical treatment of open-angle glaucoma. J Glaucoma 2016;25(7):e691-e696. DOI: 10.1097/ IJG.0000000000000352.

21. Galal A, Bilgic A, Eltanamly R, et al. XEN glaucoma implant with mitomycin C 1-year follow-up: result and complications.J Ophthalmo 2017;2017:5457246. DOI: 10.1155/2017/5457246. 